\title{
Las cesár eas en México: tendencias, niveles y factores asociados
}

\author{
Esteban Puentes-Rosas, MV, ${ }^{(1)} 0$ ctavio Gómez-D antés, MC, MSP, ${ }^{(1)}$
}

Francisco Garrido-Latorre, MC, DSP.(1)

\section{Puentes-Rosas E, Gómez-Dantés O, Garrido-Latorre F. Las cesáreas en México: tendencias, niveles y factores asociados. Salud Publica Mex 2004;46:16-22.} El texto completo en inglés de este artículo está disponible en: http://www.insp.mx/salud/index.html

\section{Resumen}

Objetivo. Describir el comportamiento de las cesáreas en México en los últimos 10 años y medir su relación con distintas variables. Material y métodos. Estudio ecológico en el que se utilizó la base de datos de nacimientos atendidos en instituciones de salud públicas y privadas que concentra la Secretaría de Salud de México. La variable dependiente fue el tipo de atención del parto. Las variables independientes fueron el producto interno bruto per cápita, el índice de desarrollo humano, el porcentaje de analfabetismo de las mujeres, el nivel de marginación y el porcentaje de médicos especialistas, entre otras. La relación entre las variables en estudio se midió con las pruebas de correlación de Pearson y de Spearman. Con los datos de 1999 se realizó un análisis de regresión lineal múltiple para identificar las principales variables asociadas con el porcentaje de cesáreas por entidad federativa. Resultados. El porcentaje de nacimientos por cesárea en el ámbito nacional se incrementó en los últimos 10 años a un ritmo ligeramente superior a $1 \%$ anual. El ritmo de crecimiento fue considerablemente mayor en la seguridad social y el sector privado. El porcentaje de cesáreas a escala nacio nal en 1999 fue ligeramente superior a 35\%. Los valores más altos correspondieron al sector privado con 53\%. Las instituciones de seguridad social presentaron un porcentaje de cesáreas de 38.2\%. Las variables con valores de asociación más intensa con el porcentaje de cesáreas fueron el porcentaje de médicos que son especialistas en el estado y el producto interno bruto per cápita. Discusión. Los resultados presentados respaldan las opiniones que defienden la implantación de políticas correctivas específicas, con el fin de impulsar un descenso en la frecuencia de cesáreas. En otros contextos se han producido

\author{
Puentes-Rosas E, Gómez-Dantés 0, \\ Garrido-Latorre F. \\ Caesarean sections in Mexico: tendencies, levels \\ and associated factors. \\ Salud Publica Mex 2004;46:16-22. \\ The English version of this paper \\ is available at: http://www.insp.mx/salud/index.html
}

(1) Dirección General de Información y Evaluación del Desempeño. Secretaría de Salud. México, DF, México.

Fecha de recibido: 27 de mayo de 2003 - Fecha de aprobado: 28 de agosto de 2003 Solicitud de sobretiros: Dr. Esteban Puentes Rosas. Reforma 450-piso 12, colonia Juárez, 06600 México, DF, México. Correo electrónico: epuentes@ salud.gob.mx 
descensos importantes con requisitos como la solicitud de una segunda o pinión antes de realizar una cesárea, la definición precisa de las razones para llevarla a cabo y el monitoreo de los porcentajes individuales de cesáreas entre los obstetras de los hospitales. El texto completo en inglés de este artículo está disponible en: http://www.insp.mx/salud/index.html

Palabras clave: cesáreas; atención del parto; índice de desarrollo humano; México
Key words: caesarean section; birth delivery; human development index; Mexico
A mediados de los años ochenta la Organización A Mundial de la Salud (OMS) propuso como estándar para los nacimientos por cesárea la cifra de $15 \% .{ }^{1}$ A pesar de esta recomendación, los nacimientos quirúrgicos se han incrementado de manera notable en los últimos años en casi todos los países de ingresos altos y medios. En España su número se duplicó en los últimos 15 años y hoy alcanza $23 \%$ de los partos. ${ }^{2}$ En el Reino Unido los nacimientos por esta vía se incrementaron de sólo 4\% en 1970 a más de 20\% en el momento actual. ${ }^{3}$ Esto obligó al Departamento de Salud a solicitarle al Colegio Real de Obstetras y Ginecólogos el más grande estudio sobre cesáreas jamás realizado en este país. En Estados Unidos de América los nacimientos quirúrgicos pasaron de 5\% en 1988 a $23 \%$ en el año 2000. ${ }^{4}$ Japón, que hace un uso muy extensivo de las parteras tradicionales, es una de las pocas naciones desarrolladas -junto con Holanda y los países escandinavos- que parece haber evitado esta epidemia. Su porcentaje de nacimientos por cesárea es menor de $10 \%$.

Las cifras de los países de ingresos medios de América Latina son todavía más alarmantes. En Brasil más de $30 \%$ de los nacimientos son cesáreas. ${ }^{5}$ En Chile este porcentaje asciende a $40 \%{ }^{6}$

Son muchas las razones que se han esgrimido para explicar este exceso de cesáreas: la percepción que tienen los prestadores de que se trata de un procedimiento más seguro que el parto vaginal; la disminución de las habilidades obstétricas del personal encargado de la atención de los partos; la creciente edad de las madres; la mayor capacidad tecnológica para detectar sufrimiento fetal; la preferencia por este procedimiento que manifiestan ciertos sectores de la sociedad, y diversos incentivos económicos relacionados con los seguros privados. ${ }^{7-11}$

La cesárea segura constituyó un importante avance en las alternativas de atención del parto. ${ }^{10}$ Hasta principios de los años sesenta, los incrementos que se presentaron en el uso de este procedimiento en los paí- ses desarrollados se asociaron con descensos en la mortalidad materna y neonatal. Cabe destacar que hasta esas fechas los porcentajes de cesáreas en estos países no superaban el 10\%. A partir de los años setenta, sin embargo, las cesáreas se incrementaron de manera muy significativa sin que se se produjeran mejoras adicionales en la mortalidad de las madres y sus productos.

El aumento de las cesáreas que no tienen justificación clínica preocupa a las autoridades sanitarias y a los prestadores de servicios de salud porque eleva los costos de la atención médica, y expone a la madre y al producto a riesgos innecesarios.

El objetivo de este trabajo es describir el comportamiento de las cesáreas en México en los últimos años, tanto en el sector público como en el privado, y evaluar su posible relación con ciertas características socioeconómicas, y la disponibilidad de especialistas.

\section{Material y métodos}

Los resultados que aquí se presentan proceden de un estudio ecológico cuyos datos se obtuvieron de los registros regulares sobre atención del parto que concentra la Dirección General de Información y Evaluación del Desempeño de la Secretaría de Salud de México (DGIED), que provienen tanto del sector público como del privado. A la base de datos creada ex profeso se le agregaron campos para ingresar datos relacionados con indicadores socioeconómicos, de desarrollo humano y de disponibilidad de prestadores de servicios de salud. La unidad de análisis fue la entidad federativa y el periodo de estudio comprendió los años 1991 a 2000. El análisis de tendencia de las cesáreas utilizó como variables el porcentaje anual de cesáreas informado por cada institución y los años comprendidos en el periodo de estudio. Estos datos se modelaron inicialmente con regresión lineal simple y posteriormente se construyó un modelo con términos de interacción por tipo de institución para probar la hipótesis nula de parale- 
lismo de las líneas de tendencia. ${ }^{12}$ Se excluyó al sector privado de este tipo de análisis puesto que no se tenían datos para todo el periodo.

Para el estudio de posibles determinantes se utilizaron los datos de 1999, año para el cual se cuenta con información para todas las variables seleccionadas. Las variables del estudio fueron las siguientes:

Variable dependiente: tipo de atención del parto (vaginal o cesárea).

Variables independientes: tipo de institución (para población no asegurada, seguridad social y privada), producto interno bruto per cápita (PIB) ${ }_{1}^{13}$ índice de desarrollo humano, ${ }^{14}$ porcentaje de analfabetismo de las mujeres, índice de marginación, ${ }^{14}$ médicos por 1000 habitantes, y médicos especialistas en ginecología y obstetricia por 1000 habitantes. Tanto el índice de desarrollo humano (IDH) como el índice de marginación son dos indicadores compuestos. El primero de ellos, desarrollado por el Programa de Naciones Unidas para el Desarrollo (PNUD) para medir el grado de desarrollo humano de los Países Miembros, incluye tres dimensiones: a) la esperanza de vida al nacer, b) el logro educativo, medido con una combinación del grado de alfabetismo de los adultos y la mediana de los años de escolaridad, y c) la capacidad de contar con acceso a los recursos que permitan disfrutar de un nivel de vida digno y decoroso, medido a través del PIB per cápita. Los valores posibles del IDH varían entre 0 y 1 , donde 1 significa el grado de desarrollo humano más alto que se puede alcanzar. El índice de marginación, desarrollado por el Consejo Nacional de Población (Conapo), incluye nueve indicadores relacionados con escolaridad, disponibilidad de servicios en la vivienda, población residente en localidades menores de 5000 habitantes y población con ingresos de hasta dos salarios mínimos. En 1999 los valores observados en este indicador por entidad federativa oscilaron entre -1.52 y 2.25 , donde -1.52 correspondió a la entidad federativa con el menor grado de marginación. ${ }^{14}$

Para seleccionar los procedimientos de análisis más adecuados se evaluó la normalidad de los datos mediante una prueba de bondad del ajuste de Kolgomorov-Smirnov. Los resultados indicaron que era posible utilizar pruebas paramétricas para evaluar las correlaciones entre las diferentes variables en todos los casos, excepto en las relaciones donde intervenían el porcentaje de analfabetismo de las mujeres y el número de especialistas en ginecología y obstetricia. Por este motivo, las correlaciones se evaluaron utilizando tanto la prueba paramétrica de Pearson como la prueba por rangos de Spearman. Se realizaron además análisis de regresión lineal en aquellos casos en los cuales la evaluación de las variables permitió concluir que este tipo de prueba era adecuada. En los casos en los que se consideró pertinente se obtuvieron los coeficientes de regresión respectivos y el valor de $R^{2}$, que expresa el grado de ajuste de la recta y el poder predictivo de la variable independiente sobre la variable de resultado. Tanto para los coeficientes de correlación como para las rectas de regresión, se consideró significativo un valor $p$ menor a 0.05 . Para las variables que mostraron asociaciones significativas se utilizó un modelo de regresión múltiple, por el método paso a paso. El análisis estadístico se llevó a cabo con el programa informático SPSS 10.0 para Windows.

\section{Resultados}

\section{Tendencia de los nacimientos por cesárea}

Como se puede observar en la figura 1, en 1991 las tasas de cesáreas en el país ya se encontraban elevadas. El porcentaje de nacimientos por cesárea se incrementó en los últimos 10 años a un ritmo ligeramente superior a $1 \%$ anual. Sin embargo, el incremento en el porcentaje de cesáreas ha tenido un comportamiento diferente en las distintas instituciones que prestan servicios de salud. Mientras que en los servicios prestados por el IMSSSolidaridad* el porcentaje de nacimientos por cesárea se ha mantenido relativamente estable, con un incremento que no ha llegado siquiera a un cuarto de punto porcentual por año, en el Instituto de Seguridad y Servicios Sociales de los Trabajadores del Estado (ISSSTE) y el sector privado el incremento ha sido, en promedio, superior a los dos puntos porcentuales por año. El análisis del paralelismo en las rectas ajustadas a partir de las observaciones en los porcentajes de cesáreas por institución mostró que la diferencia entre éstas es estadísticamente significativa $(p<0.01)$.

\section{Porcentaje actual de nacimientos por cesárea}

El porcentaje nacional de cesáreas en 1999 fue ligeramente superior a $35 \%$. Los porcentajes de nacimientos atendidos por cesárea variaron de manera importante entre los diferentes estados, pero en todos los casos fueron superiores a $15 \%$, porcentaje que, como ya se señaló, la OMS recomienda como límite superior. En

*Ahora, IMSS-0 portunidades 
los estados el valor máximo en el porcentaje de cesáreas fue el de Nuevo León, con $49.89 \%$. El valor mínimo correspondió a Zacatecas, con $24.86 \%$. La cuarta parte de las entidades mostró valores por arriba de $36 \%$. Los valores correspondientes a cada uno de los estados en 1999 se muestran en la figura 2.

\section{Cesáreas en los diferentes subsistemas de salud}

Las cifras de cesáreas de las distintas instituciones de salud variaron considerablemente. Los valores más altos correspondieron al sector privado con 53\%. Las instituciones de seguridad social (IMSS, ISSSTE) presentaron un porcentaje de cesáreas de 38.2\%. Finalmente, en las instituciones dedicadas a atender a la población no asegurada -Secretaría de Salud (SSA), IMSS-Solidaridad-el porcentaje de cesáreas en 1999 ascendió a 24.8\%. El valor más alto entre las instituciones públicas se alcanzó en el ISSSTE $(50.7 \%)$ y el más bajo correspondió a los servicios del sistema IMSS-Solidaridad (17.5\%). Los valores correspondientes a las diversas instituciones de salud en 1999 se muestran en la figura 3.

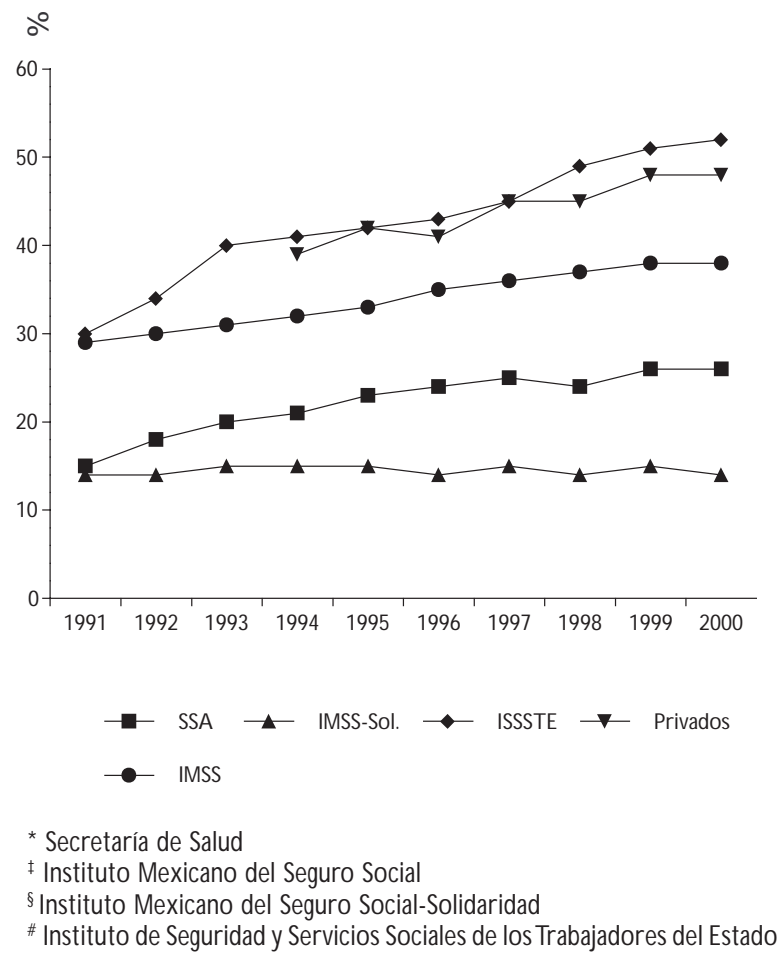

Figura 1. Porcentaje de nacimientos por cesárea en DiVerSAS INSTITUCIONES DE SALUd. MÉXICO, 1991-2000

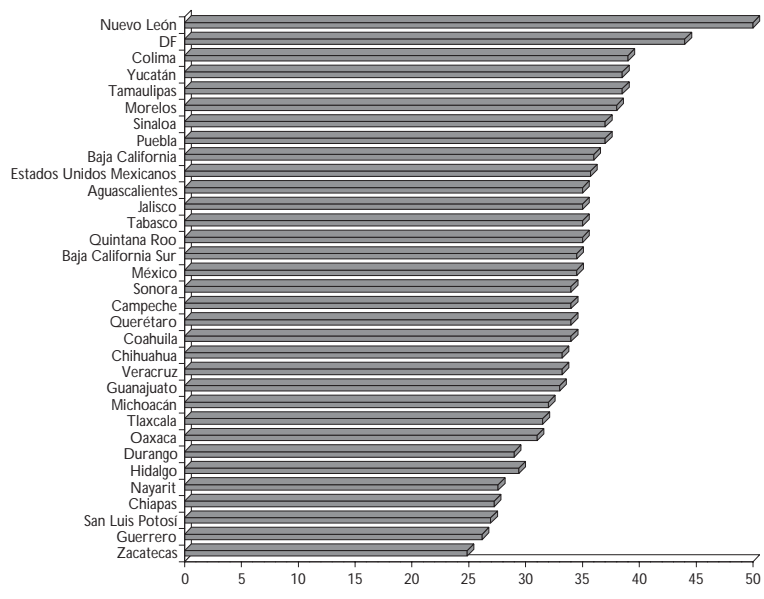

Figura 2. Porcentaje estatal de nacimientos por Cesárea. México, 1999

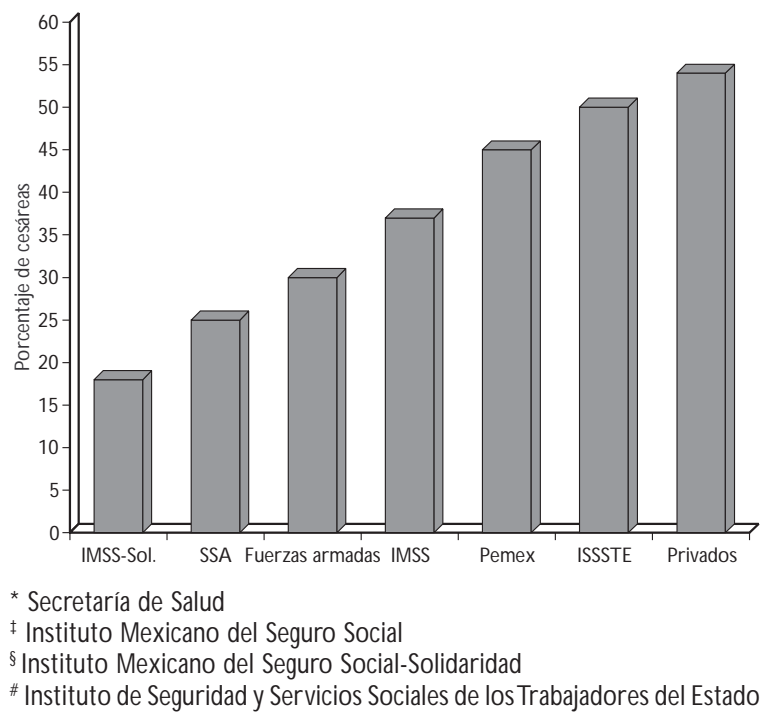

Figura 3. Porcentaje de nacimientos por cesárea en DiVERSAS INSTITUCIONES DE SALUd. MÉXICO, 1999

\section{Cesáreas en el sector privado}

A escala nacional, el porcentaje de cesáreas en el sector privado fue de $53 \%$. El análisis comparativo de la distribución de los datos correspondientes a los estados en general, y al sector privado en particular, permiten concluir que las diferencias entre estados en este sector son menos pronunciadas que cuando se utilizan cifras globales. Más de $25 \%$ de los estados mostró valores superiores a 56\%. En Nuevo León el porcentaje de cesáreas en los servicios privados alcanzó la cifra de $73.6 \%$. 
Para determinar una posible asociación entre el porcentaje de cesáreas y la cantidad de nacimientos atendidos, se realizó una prueba de correlación por rangos de Spearman, y se encontró un valor del coeficiente de correlación de 0.005 que indica que el porcentaje de cesáreas no guarda ninguna correlación con la cantidad de partos atendidos en las diferentes unidades privadas estudiadas.

\section{Variables asociadas con el porcentaje de cesáreas realizadas en los estados}

Al elaborar los análisis de correlación se observaron asociaciones intensas con ciertas variables y moderadas con algunas otras. Las correlaciones fueron evaluadas mediante la prueba paramétrica de Pearson, la prueba por rangos de Spearman y con análisis de regresión lineal simple. Las variables con valores de asociación más intensa con el porcentaje de cesáreas fueron el porcentaje de médicos en el estado que son especialistas, el PIB per cápita y el IDH. Los resultados del análisis de correlación y los coeficientes de la regresión lineal simple con algunas variables se muestran en el cuadro I.

Cuadro I

\section{Coeficientes de correlación y de Regresión entre EL PORCENTAJE ESTATAL DE CESÁREAS Y VARIABLES SELECCIONADAS, MÉXICO, 1999}

\begin{tabular}{|c|c|c|c|}
\hline $\begin{array}{l}\text { Coef } \\
\text { cor } \\
\text { de }\end{array}$ & $\begin{array}{l}\text { eficiente de } \\
\text { correlación } \\
\text { de Pearson }\end{array}$ & $\begin{array}{l}\text { Coeficiente } \\
\text { de regresión }\end{array}$ & $\begin{array}{l}\text { Intervalo de confianza } \\
(95 \%) \text { para el } \\
\text { coeficiente de regresión }\end{array}$ \\
\hline $\begin{array}{l}\text { Proporción del } \\
\text { personal } \\
\text { médico que son } \\
\text { especialistas }\end{array}$ & $\begin{array}{r}0.665 \\
p<0.001\end{array}$ & 71.04 & $40.7,101.4$ \\
\hline Indice de marginación & $\begin{array}{r}-0.476 \\
p=0.006\end{array}$ & -2.43 & $-4.10,-0.75$ \\
\hline $\begin{array}{l}\text { Proporción de } \\
\text { municipios calificados } \\
\text { como no marginados }\end{array}$ & $p=0.006$ & 7.89 & $2.60,13.17$ \\
\hline $\begin{array}{l}\text { Porcentaje de } \\
\text { analfabetas }\end{array}$ & $\begin{array}{r}-0.508 \\
p=0.003\end{array}$ & -0.478 & $-0.775,-0.181$ \\
\hline $\begin{array}{l}\text { Producto interno } \\
\text { bruto per cápita }\end{array}$ & $\begin{array}{r}0.646 \\
p<0.001\end{array}$ & 0.00076 & $0.000,0.001$ \\
\hline $\begin{array}{l}\text { Indice de desarrollo } \\
\text { humano }\end{array}$ & $\begin{array}{r}0.653 \\
p<0.001\end{array}$ & 80.45 & $46.30,114.61$ \\
\hline Mortalidad hebdomadal & dal $\begin{array}{r}0.360 \\
p=0.046\end{array}$ & 0.972 & $0.037,1.907$ \\
\hline $\begin{array}{l}\text { Proporción de la } \\
\text { población estatal con } \\
\text { derechohabiencia }\end{array}$ & $\begin{array}{r}0.579 \\
p=0.001\end{array}$ & 20.06 & $9.52,30.60$ \\
\hline
\end{tabular}

\section{Regresión lineal múltiple}

Por último, se ajustó un modelo lineal multivariado eliminando del modelo aquellas variables que mostraron correlaciones intensas entre sí. El mejor modelo ajustado fue el que contó con dos variables específicas: la proporción del personal médico que es especialista y el PIB per cápita estatal. El coeficiente de determinación $\left(R^{2}\right)$ de la ecuación fue de 0.54 , lo que significa que poco más de la mitad de la variación en los porcentajes de cesáreas en los estados puede estar explicada por las diferencias en estas dos variables. El valor del estadístico $F$ del modelo fue de 15.63, con un valor $p=0.001$. Los coeficientes correspondientes al modelo final y su significancia estadística se presentan en el cuadro II.

\section{Discusión}

Los resultados que se presentan en este trabajo muestran: a) que las cesáreas en México se han incrementado considerablemente en los últimos 10 años, en particular en las instituciones de la seguridad social y en el sector privado; b) que los niveles alcanzados superan con mucho el estándar fijado por la OMS; c) que este incremento está asociado con el nivel socioeconómico de las entidades federativas, y d) que la oferta de servicios de especialidad también parece jugar un papel importante en el crecimiento explosivo reciente de este procedimiento.

Los nacimientos por cesárea en México han tenido un comportamiento similar al de la mayoría de los países europeos y de América del Norte, y al de los países de ingresos medios de América Latina. El porcentaje de incremento de las cesáreas en nuestro país en los últimos 10 años ha sido de poco más de $1 \%$ anual, pero en

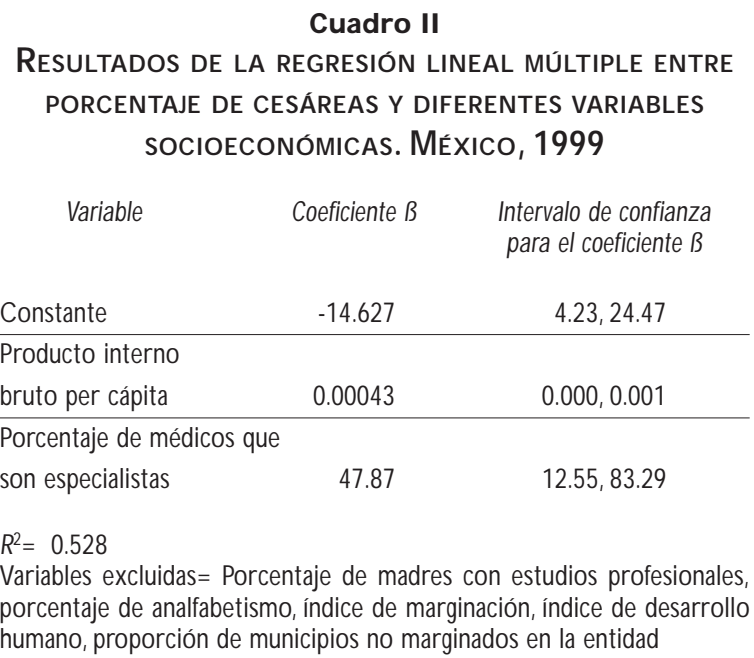

salud pública de méxico / vol.46, no.1, enero-febrero de 2004 
algunas instituciones públicas (IMSS, ISSSTE) y en el sector privado la tendencia al alza es todavía mayor.

Este incremento reciente ha colocado el porcentaje nacional de cesáreas en poco más de 35\%, cifra comparable a la de Brasil y Chile. ${ }^{5}$ Este porcentaje podría resultar aceptable en unidades que concentran embarazos de alto riesgo, pero en el ámbito nacional no hay ningún factor -altas prevalencias de diabetes e hipertensión arterial o alteraciones del crecimiento de los productos- que pudiera justificar una cifra tan elevada de partos quirúrgicos. De hecho, en un estudio realizado en el IMSS se concluyó que un alto porcentaje de las cesáreas llevadas a cabo en esa institución estaban poco justificadas desde el punto de vista médico. ${ }^{15}$

La única variable que parecería justificar este aumento son las cesáreas previas. Una nueva intervención quirúrgica en estos casos evita, según algunos investigadores, una ruptura uterina. ${ }^{16,17} \mathrm{Sin}$ embargo, hay datos que permiten concluir que, salvo en situaciones muy específicas, las cesáreas repetidas no incrementan la seguridad ni confieren beneficios adicionales. ${ }^{18,19}$

Así, si tomamos como punto de referencia la cifra de $15 \%$ propuesta por la OMS, podríamos concluir que en 1999 se realizaron en México 361329 cesáreas más de las debidas.

La relación directa entre el porcentaje de cesáreas y los niveles de ingreso que se muestran en los resultados de este trabajo se han observado en otros contextos nacionales. En Brasil y Chile la probabilidad de cesárea es mayor cuando un nacimiento se atiende en clínicas privadas y la madre pertenece a estratos de ingresos medios y altos. ${ }^{5,6,20,21}$ En esta relación influyen múltiples factores, pero destacan dentro de ellos los incentivos financieros, tanto para los proveedores directos del servicio como para los hospitales, y la preferencia que por razones de estatus o seguridad, entre otras, manifiestan las mujeres de las clases media y alta por este tipo de procedimiento. En México, Campero y colaboradores han sugerido que algunos ginecobstetras, atraídos sobre todo por beneficios económicos y con argumentos de una mayor seguridad y menores efectos secundarios, han creado una demanda de cesáreas entre las mujeres de los grupos socioeconómicos más favorecidos. ${ }^{22}$

La "demanda inducida" por lo general se asocia a una falta de información entre las usuarias de servicios de maternidad y a una muy poca participación de las madres en las decisiones relacionadas con los nacimientos. Esto ha llevado a diversos grupos de consumidores a pugnar, ya no tanto por un "consentimiento informado" como lo que se ha denominado "elección informada": una elección basada en la comprensión in- tegral de todas las opciones y de sus posibles riesgos y beneficios. $^{23}$

Finalmente, este trabajo también demuestra que, al igual que en otros ámbitos geográficos, en México la disponibilidad de especialistas está estrechamente ligada con la frecuencia de las cesáreas. Hay autores que sugieren que este hecho está relacionado con una creciente disponibilidad de mejor tecnología quirúrgica que los especialistas "no pueden dejar de utilizar", en contraste con los médicos generales y familiares, que no están capacitados para hacer uso de ella. ${ }^{24}$ Esta tecnología, además, es promocionada de manera muy agresiva por la industria.

El perfil del especialista juega también un papel central. Muchos obstetras, formados en ambientes cada vez más intervencionistas, están mejor capacitados para atender partos quirúrgicos que para atender nacimientos que podrían efectuarse de manera segura por vía vaginal. ${ }^{25}$ Berkowitz y colaboradores también encontraron que los médicos jóvenes tienen mayor probabilidad de llevar a cabo una cesárea cuando enfrentan problemas de distocia que los médicos más experimentados. ${ }^{26}$

A reserva de profundizar en las causas del incremento explosivo de las cesáreas en México para diseñar políticas correctivas específicas, parece razonable pugnar por un descenso generalizado de este procedimiento. En nuestro país se cuenta por lo menos con el reporte de una experiencia en el Hospital Civil de Guadalajara que permitió disminuir el porcentaje de cesáreas de 28 a $13 \%$ en un lapso de cinco años. ${ }^{27}$ Las medidas adoptadas no sólo no tuvieron consecuencias negativas en la mortalidad materna sino que produjeron una disminución moderada pero significativa de la mortalidad neonatal. En otros contextos se han tenido resultados similares con la implantación de medidas tan simples como el requisito de una segunda opinión antes de realizar una cesárea, la definición precisa de las razones para llevarla a cabo y el monitoreo de los porcentajes individuales de cesáreas entre los obstetras de los hospitales. $^{28,29}$

Deben hacerse algunas puntualizaciones respecto al alcance de este trabajo. Como todos los estudios ecológicos, éste no permite identificar cómo se distribuyen las variables de interés en el nivel individual. Así, por ejemplo, el hecho de que haya más cesáreas en las entidades con mayor PIB per cápita no puede garantizar que sean las mujeres de mayores ingresos las que estén recurriendo de manera más intensa al nacimiento por cesárea, aunque en realidad ésta es una hipótesis plausible. Por otro lado, los registros regulares de información sobre la atención al parto pueden tener una calidad diferente en los distintos ámbitos de atención. Por ejemplo, el sector privado apenas comienza a incorporarse 
en el sistema regular de información en salud, por lo que los registros correspondientes a este sector podrían carecer de algunas características deseables. De cualquier modo, los datos con que se cuenta nos hablan de un claro incremento de las cesáreas en México, y las cifras analizadas y presentadas podrían considerarse como un escenario mínimo del fenómeno.

\section{Referencias}

1. W orld Health 0 rganization. A ppropriate technology for birth. Lancet 1985;2:436-437.

2. Cerda H. Uno de cada cuatro niños nace por cesárea. El País 2001; octubre 30:28.

3. Johanson $\mathrm{R}, \mathrm{N}$ ewburn $\mathrm{M}, \mathrm{Macfarlane} \mathrm{A}$. Has the medicalisation of childbirth gone too far? BMJ 2002;324:892-895.

4. Martin JA, Hamilton BE,Ventura SJ, Menacker F, Park MM. Births: Final data for 2000. N atl Vital Stat Rep 2002;(5):1-101.

5. Belizán JM,Althabe F, Barros FC, A lexander S. Rates and implications

of caesarean sections in Latin America: Ecological study. BMJ

1999;319:1397-1402.

6. Murray SF. Relation between private health insurance and high rates of caesarean section in Chile: Q ualitative and quantitative study. BMJ 2000;321:1501-1505.

7. Amu 0, Rajendran S, Bolaji I. Maternal choice alone should not determine method of delivery. BMJ 1998;317:462-465.

8. Sultan AH, Kamm MA, Hudson CN, Thomas JM, Bartram Cl.A sphincter disruption during vaginal delivery. N Engl J Med 1993;329:1905-1911.

9. Al-M ufti R, McC arthy A, Fisk N M. O bstetricians' personal choice and mode of delivery. Lancet 1996;347:544.

10. De Costa C.A sort of progress. Lancet 1998;351:1202-1203.

11. Payne D. 0 reland sees big increase in caesarean sections. BM] 2000;320:140.

12. Kleinbaum D, Kupper L, Muller K.Applied regression analysis and other multivariable methods. Second edition, Belmont: D uxbury Press, 1988.
13. Instituto N acional de Estadística, Geografía e Informática. Sistema de Cuentas N acionales. PIB por entidad federativa 1993-2000. A guascalientes, México: IN EGI, 2000.

14. Consejo $\mathrm{N}$ acional de Población. México en cifras. Disponible en: www.conapo.gob.mx/m_en_cifras/principal.html. Consultado el 15 de marzo de 2002.

15.Velasco V, N avarrete E, Pozos JL, 0 jeda RI, Cárdenas C, C ardona JA. Indicaciones y justificación de las cesáreas en el IMSS. Gac Med Mex 2000;136:421-431.

16. Placek PJ,Taffel SM. Recent patterns in cesarean delivery in the United States. 0 bstet Gynecol 1985;28:735-744.

17. Taffel SM, Placek PJ, Liss T.Trends in the U nited States cesarean section rate and reasons for the 1980-85 rise. Am J Public Health 1987;77:955-959.

18. Flamm BL.Vaginal birth after cesarean section: Controversies old and new. C O bstet Gynecol 1985;28:735-744.

19. Placek 0 J,Taffel SM.Vaginal birth after cesarean section in the 1980. Am J Public Health 1988;78:512-515.

20. Barros FC, Vaughan JP,Victora CG, Huttly RS. Epidemic of caesarean sections in Brazil. Lancet 1991;338:167-169.

21. Béhague D P,Victora CG, Barros FC. C onsumer demand for caesarean sections in Brazil: Population based birth cohort study linking ethnographic and epidemiological methods. BMJ 2002;324:942-945.

22. C ampero L, García C, Díaz C, 0 rtiz 0, Reynoso S, Langer A. "Alone, I wouldn't have known what to do":A qualitative study on social support during labor and delivery in Mexico. Soc Sci Med 1998;47:395403.

23. Paterson-Brown S.Yes, as long as the woman is fully informed. BMJ 1998:317:462-465

24. Groopman J.A knife in the back. N ew Yorker 2002;A pril 8:66-73.

25. Goyert G, Bottoms S, Treadwell M, N ehra P.The physician factor in caesarean birth rates. N Engl J Med 1989;320:706-709.

26. Berkowitz GS, Fiarman GS, Mojica MA.The effect of physician characteristics on the caesarean birth rates.Am J O bstet Gynecol 1989;161:146-149.

27. Guzmán S. La reducción de cesáreas de 28 a 13\% incrementa o no la mortalidad materna y perinatal: la gran pregunta. Ginecol 0 bstet Mex 1993;66:122-125.

28. Myers S, G leicher N .A succesful program to lower caesarean section rates. N Engl J Med 1988;319:1511-1516.

29. Flamm BL, Berwick D M, Kabcenell A. Reducing caesarean section rates safely: Lessons from a "breakthrough series" collaborative. Birth 1998;25(2):117-124. 